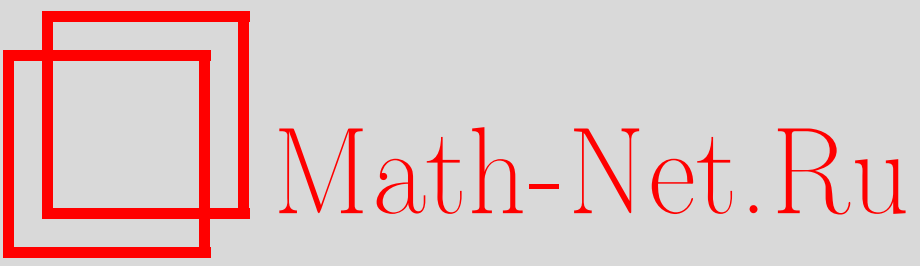

В. В. Пупышев, K задаче трех частиц с парными взаимодействиями, обратно пропорциональными квадратам расстояний, ТМФ, 2001, том 128, номер 2, 268 287

DOI: https://doi.org/10.4213/tmf496

Использование Общероссийского математического портала Math-Net.Ru подразумевает, что вы прочитали и согласны с пользовательским соглашением http://www . mathnet.ru/rus/agreement

Параметры загрузки:

IP : 54.89 .56 .158

26 апреля 2023 г., $14: 41: 52$ 


\section{К ЗАДАЧЕ ТРЕХ ЧАСТИЦ С ПАРНЫМИ ВЗАИМОДЕЙСТВИЯМИ, ОБРАТНО ПРОПОРЦИОНАЛЬНЫМИ КВАДРАТАМ РАССТОЯНИЙ}

Метод гипергармоник применяется для исследования трехчастичных уравнений Шредингера и Фаддеева с парными взаимодействиями, обратно пропорциональными квадратам расстояний. Точные решения таких уравнений строятся в виде произведения зависящей от гиперрадиуса функции Бесселя на конечную линейную комбинацию гипергармоник. Доказывается и исследуется критерий существования таких решений.

\section{1. ВВЕДЕНИЕ}

Радиальное двухчастичное уравнение Шредингера с потенциалом $v(x)=c x^{-2}$ и угловым моментом $b$ при положительной энергии $e$ и достаточно большой константе $c$,

$$
e \geqslant 0, \quad c>c_{b} \equiv-\left(b+\frac{1}{2}\right)^{2}
$$

имеет только сингулярное на малых расстояниях $x$ решение $\psi^{b}$ (см., например, [1]). Это решение физически допустимо $\left(x \psi^{b} \rightarrow 0, x \rightarrow 0\right)$ :

$$
\psi^{b}(x ; e) \sim O\left(x^{q}\right) \rightarrow 0, \quad q \equiv-\frac{1}{2}+\sqrt{c+c_{b}}>0, \quad x \rightarrow 0 .
$$

Функция $\psi^{b}$ описывает рассеяние, причем при $c<c_{b}$ имеется и дискретный спектр, а основное состояние отвечает энергии $e=-\infty$. В этом состоянии частицы находятся в бесконечно малой окрестности их центра масс $(x=0)$, поэтому говорят, что происходит "падение" частиц в точку $x=0$.

Взаимодействие, обратно пропорциональное квадрату расстояния, часто называют взаимодействием центробежного типа. В обшем случае теорема сушествования и единственности физически приемлемых решений задачи трех и более частиц с парными взаимодействиями центробежного типа либо с парными взаимодействиями, ведущими себя

* Объединенный институт ядерных исследований, г. Дубна Московской обл., Россия. E-mail: pupyshev@thsun1.jinr.ru 
как такие взаимодействия лишш при малых или же больших расстояниях, не доказана [2]. Некоторые приемлемые решения такой задачи и достаточные для их сушествования условия найдены лишь в частном случае [3], а именно: в задаче трех тождественных частиц с $S$-волновыми парными взаимодействиями центробежного типа и полным угловым моментом, равным (см. [4]) или не обязательно равным (см. [5]) нулю.

В предыдущей работе [5] был предложен метод построения и доказательства критерия существования точных решений задачи трех тождественных частиц. Эти решения представимы в виде произведения зависящей от гиперрадиуса функции Бесселя на конечную линейную комбинацию трехчастичных гипергармоник. Данный метод является аналитическим и достаточно простым, так как в его рамках исходная задача трех тождественных частиц сводится к анализу на совместность и последуюшему решению конечных систем линейных уравнений. Поэтому и для дальнейшего развития теории Фаддеева, и для прикладных аналитических исследований представляется интересным обобщение этого метода для систем из трех не обязательно тождественных частиц с центральными парными взаимодействиями центробежного типа или же с конечными подсуммами операторных разложений таких взаимодействий по их собственным базисам из сферических функций. Такое обобщение - главная цель настоящей работы.

\section{2. ОСНОВНЫЕ ПОНЯТИЯ И ОБОЗНАЧЕНИЯ}

Символом $\left(p_{1}, p_{2}, p_{3}\right)$ обозначим исследуемую систему трех $p a з н ы x$ частиц $p_{1}, p_{2}$ и $p_{3}$ с массами $m_{1}, m_{2}$ и $m_{3}$. Стандартным образом [6] в ее шестимерном координатном пространстве $\mathcal{R}^{6}$ определим приведенные координаты Якоби $\mathbf{x}_{i}, \mathbf{y}_{i}, i=1,2,3$. Объединим их в шестимерные векторы $\mathbf{r}_{i}=\left(\mathbf{x}_{i}, \mathbf{y}_{i}\right)$ с обычными гиперсферическими координатами $\left(r, \Omega_{i}\right)[7]$, где $r \equiv\left(x_{i}^{2}+y_{i}^{2}\right)^{1 / 2}$ - гиперрадиус, а $\Omega_{i} \equiv\left(\hat{x}_{i}, \hat{y}_{i}, \varphi_{i}\right)$ - набор из пяти гиперсферических углов: $\hat{x}_{i}$ и $\hat{y}_{i}-$ пары сферических углов векторов $\mathbf{x}_{i}$ и $\mathbf{y}_{i}, \operatorname{a~} \varphi_{i} \equiv \operatorname{arctg}\left(y_{i} / x_{i}\right)$. В $\mathcal{R}^{6}$ сушествуют три $(i=1,2,3)$ декартовых $\left(\left\langle\mathbf{r}_{i}\right|=\left\langle\mathbf{x}_{i}, \mathbf{y}_{i}\right|\right)$ и три соответствующих им гиперсферических $\left(\left\langle\mathbf{r}_{i}\right|=\left\langle r_{i}, \Omega_{i}\right|\right)$ координатных представления. Переход от представления $\left\langle\mathbf{r}_{i}\right|$ к другому представлению $\left\langle\mathbf{r}_{k}\right|, k \neq i$, называется кинематическим преобразованием (см. [3]) и характеризуется кинематическим углом

$$
\gamma_{k i} \equiv g_{k i} \operatorname{arctg}\left(\frac{m_{j}\left(m_{1}+m_{2}+m_{3}\right)}{m_{k} m_{i}}\right)^{\frac{1}{2}} .
$$

По определению таких шести углов, если $(k, i)=(1,2),(3,1),(2,3)$, то

$$
g_{k i}=-g_{i k}=1, \quad 0 \leqslant \gamma_{k i} \leqslant \frac{\pi}{2}, \quad \sum_{(k, i)} \gamma_{k i}=\pi,
$$

а значения $\gamma_{k i}= \pm \pi / 2, \pm \pi / 4,0$ являются предельными. Например,

$$
\begin{array}{llll}
\gamma_{12} \rightarrow \frac{\pi}{2}, & \gamma_{23} \rightarrow 0, \quad \gamma_{31} \rightarrow \frac{\pi}{2}, & \text { если } & \frac{m_{1}}{m_{2}} \rightarrow 0, \quad m_{2}=O\left(m_{3}\right) ; \\
\gamma_{12} \rightarrow \frac{\pi}{4}, & \gamma_{23} \rightarrow \frac{\pi}{2}, \quad \gamma_{31} \rightarrow \frac{\pi}{4}, & \text { если } & \frac{m_{1}}{m_{2}} \rightarrow \infty, \quad m_{2}=m_{3} .
\end{array}
$$


Пусть $I$ - единичный оператор, $\mathbf{l}_{x_{i}}$ и $\mathbf{l}_{y_{i}}$ - операторы угловых моментов, сопряженные векторам $\mathbf{x}_{i}$ и $\mathbf{y}_{i}, \mathbf{a} \mathbf{l} \equiv \mathbf{l}_{x_{i}}+\mathbf{l}_{y_{i}}$ и $\mathbf{L}-$ операторы полного углового момента и гипермомента. Квадраты всех перечисленных операторов и оператор инверсии $\mathbf{r}_{i} \rightarrow-\mathbf{r}_{i}$ коммутируют с оператором кинематического преобразования $K(\gamma)$ [8]. Если $\gamma=\gamma_{k i}$, то $K\left(\gamma_{k i}\right)=P_{i k} P_{j k}$, где $P_{i j}$ - оператор перестановки частиц $p_{i}$ и $p_{j}$.

Свободный гамильтониан $H_{0}$ системы $\left(p_{1}, p_{2}, p_{3}\right)$ в представлении $\left\langle\mathbf{r}_{i}\right|=\left\langle r, \Omega_{i}\right|$ имеет Вид

$$
H_{0}\left(\mathbf{r}_{i}\right)=-r^{-5} \partial_{r}\left(r^{5} \partial_{r}\right)+r^{-2} \mathbf{L}^{2}\left(\Omega_{i}\right) .
$$

Для каждой пары $\left(p_{i}, p_{j}\right)$ частиц $p_{i}$ и $p_{j}$ определим оператор взаимодействия $V_{k}(k=$ $1,2,3)$ в его собственном декартовом представлении $\left\langle\mathbf{r}_{k}\right|:$

$$
V_{k}\left(\mathbf{x}_{k}\right) \equiv c_{k} x_{k}^{-2} I_{k}^{d}, \quad I_{k}^{d} \equiv \sum_{b=0}^{d} P_{k}^{b}, \quad P_{k}^{b} \equiv \sum_{\beta=-b}^{b}\left|Y_{b \beta}\left(\hat{x}_{k}\right)\right\rangle\left\langle Y_{b \beta}\left(\hat{x}_{k}\right)\right|
$$

где $c_{k}$ - ненулевая действительная константа, индекс $b$ принимает целые значения, причем $b \leqslant d$, а $Y_{b \beta}$ - сферическая функция. Если $b$ пробегает все значения от 0 до $\infty$, т.е. $d=\infty$, то $I_{k}^{d}$ - единичный оператор: $I_{k}^{d}=I$. Действительно, тогда $I_{k}^{d}-$ сумма всех проекторов $P_{k}^{b}$ на состояния частиц $p_{i}$ и $p_{j}$ с угловыми моментами $\ell_{x_{k}}=b$. В этом случае $V_{k}$ - центральное взаимодействие $\left(V_{k}\left(\mathbf{x}_{k}\right)=V_{k}\left(x_{k}\right)\right)$, действуюшее в его собственном гиперсферическом представлении $\left\langle\mathbf{r}_{k}, \Omega_{k}\right|$ как оператор умножения на функцию $c_{k} /\left(r^{2} \cos ^{2} \varphi_{k}\right)$. В любом другом случае взаимодействие $V_{k}$ - нецентральное, так как является подсуммой операторного разложения центрального взаимодействия $V_{k}\left(x_{k}\right)$ по его собственному базису из сферических функций и включено лиш в определенном числе парциальных волн. Например, при $b=d=0$ это взаимодействие является $S$-волновым. В несобственном представлении $\left\langle r_{i}, \Omega_{i}\right|, i \neq k$, оператор $V_{k}$ становится более сложным, так как функция $\cos ^{2} \varphi_{k}$ зависит от углов $\Omega_{i}$ и $\gamma_{k i}$ :

$$
\begin{gathered}
V_{k}\left(\mathbf{x}_{k}\left(\mathbf{r}_{i} ; \gamma_{k i}\right)\right)=I_{k}^{d} \frac{c_{k}}{r^{2} \cos ^{2} \varphi_{k}}, \quad \varphi_{k}=\varphi_{k}\left(\varphi_{i}, u, \gamma_{k i}\right) \\
\cos ^{2} \varphi_{k}=\cos ^{2}\left(\gamma_{k i}-\varphi_{i}\right)+\frac{1}{2}(u-1) \sin 2 \gamma_{k i} \sin 2 \varphi_{i}, \quad u \equiv \frac{\mathbf{x}_{i} \cdot \mathbf{y}_{i}}{x_{i} y_{i}}
\end{gathered}
$$

Состояние $\langle\varepsilon|$ системы $\left(p_{1}, p_{2}, p_{3}\right)$ характеризуется полным набором $\varepsilon$ сохраняюшихся квантовых чисел. При любых индексах $b$ и $d$ в представлениях взаимодействий $(7)$ сохраняется полная энергия $E$ этой системы, квадрат $\ell(\ell+1)$ ее полного углового момента 1 и его третья проекция $m$, т.е. $E, \ell, m \in \varepsilon$. Если в этих представлениях $b=$ $0,1, \ldots, d=\infty$ или же $b$ принимает только четные или только нечетные значения, то в силу соотношения $\left(P_{i j}-I\right) V_{k}\left(x_{k}\right)=0$ или соответственно $P_{i j} Y_{b \beta}\left(\hat{x}_{k}\right)=(-1)^{b} Y_{b \beta}\left(\hat{x}_{k}\right)$ сохраняется и четность $\sigma$ по отношению к инверсии $\mathbf{r}_{i} \rightarrow-\mathbf{r}_{i}$. В этих трех случаях $\varepsilon=(E, \ell, m, \sigma)$.

Решение $\Psi^{\varepsilon}$ уравнения Шредингера для трехчастичного состояния $\langle\varepsilon|$

$$
\left(H_{0}+\sum_{k=1}^{3} V_{k}-E\right) \Psi^{\varepsilon}=0
$$


и сопоставленное этому решению формулой

$$
\Psi^{\varepsilon}=\sum_{k=1}^{3} \Psi_{k}^{\varepsilon}
$$

решение $\left(\Psi_{1}^{\varepsilon}, \Psi_{2}^{\varepsilon}, \Psi_{3}^{\varepsilon}\right)$ системы уравнений Фаддеева [2]

$$
\left(H_{0}-E\right) \Psi_{i}^{\varepsilon}=-V_{i} \sum_{k=1}^{3} \Psi_{k}^{\varepsilon}, \quad i=1,2,3
$$

будем искать в классе $\mathcal{B}^{\varepsilon}$, состоящем из квадратично интегрируемых на единичной сфеpe $\mathcal{S}^{5}$ в $\mathcal{R}^{6}$ функций, обладающих данным набором квантовых чисел $\varepsilon$. Для такого класса вполне определенные трехчастичные гипергармоники [6]

$$
\begin{gathered}
Y_{L a b}^{\ell m}\left(\Omega_{i}\right)=N_{L a b}\left(\sin \varphi_{i}\right)^{a}\left(\cos \varphi_{i}\right)^{b} P_{n}^{\left(a+\frac{1}{2}, b+\frac{1}{2}\right)}\left(\cos 2 \varphi_{i}\right) \mathcal{Y}_{a b}^{\ell m}\left(\hat{y}_{i}, \hat{x}_{i}\right), \\
L=a+b+2 n, \quad n=0,1, \ldots, \quad \mathbf{a}+\mathbf{b}=1,
\end{gathered}
$$

образуют полный и ортонормированный на $\mathcal{S}^{5}$ угловой базис. Здесь $N_{L a b}$ - известньй нормировочный множитель, $P_{n}^{(a+1 / 2, b+1 / 2)}-$ полином Якоби и $\mathcal{Y}_{a b}^{\ell m}-$ бисферическая гармоника. В случае $\sigma \in \varepsilon$ индексы $a$ и $b$ подчиняются еще одному дополнительному условию: $(-1)^{a+b}=\sigma$.

Гипергармоники $(13)$ - собственные функции операторов $P_{j k}, \mathbf{l}_{x_{i}}^{2}, \mathbf{l}_{y_{i}}^{2}$ и $\mathbf{L}^{2}$ :

$$
\begin{aligned}
\left(P_{j k}-(-1)^{b}\right) Q & =0, \quad\left(\mathbf{l}_{y_{i}}^{2}-a(a+1)\right) Q=0 \\
\left(\mathbf{l}_{x_{i}}^{2}-b(b+1)\right) Q & =0, \quad\left(\mathbf{L}^{2}-L(L+4)\right) Q=0, \quad Q \equiv Y_{L a b}^{\ell m}\left(\Omega_{i}\right),
\end{aligned}
$$

но не являются таковыми для оператора $K\left(\gamma_{k i}\right)$. Этот оператор сохраняет квантовые числа $L, \ell(\ell+1), m, \sigma$, но не сохраняет числа $a(a+1)$ и $b(b+1)$ :

$$
K\left(\gamma_{k i}\right) Y_{L a^{\prime} b^{\prime}}^{\ell m}\left(\Omega_{i}\right) \equiv Y_{L a^{\prime} b^{\prime}}^{\ell m}\left(\Omega_{k}\left(\Omega_{i} ; \gamma_{k i}\right)\right)=\sum_{a b}\left\langle a b\left|K\left(\gamma_{k i}\right)\right| a^{\prime} b^{\prime}\right\rangle_{L \ell} Y_{L a b}^{\ell m}\left(\Omega_{i}\right)
$$

Здесь и далее, если не оговорено специально, индексы $a$ и $b$ пробегают все допустимые при заданных $L$ и $\varepsilon$ значения. Матричные элементы оператора $K(\gamma)$ в случае $\gamma=\gamma_{k i}$ являются коэффициентами Рейнала-Реваи [9] и при любых $\gamma, \gamma^{\prime} \in[-\pi / 2, \pi / 2]$ подчиняются следуюшим соотношениям симметрии, ортонормированности и правилу сумм [8]:

$$
\begin{gathered}
\left\langle a^{\prime} b^{\prime}|K(-\gamma)| a b\right\rangle_{L \ell}=(-1)^{b^{\prime}+b}\left\langle a^{\prime} b^{\prime}|K(\gamma)| a b\right\rangle_{L \ell}, \\
\sum_{a b}\left\langle a^{\prime} b^{\prime}|K(\gamma)| a b\right\rangle_{L \ell}\left\langle a b|K(-\gamma)| a^{\prime \prime} b^{\prime \prime}\right\rangle_{L \ell}=\delta_{a^{\prime} a^{\prime \prime}} \delta_{b^{\prime} b^{\prime \prime}}, \\
\left\langle a^{\prime} b^{\prime}\left|K\left(\gamma+\gamma^{\prime}\right)\right| a^{\prime \prime} b^{\prime \prime}\right\rangle_{L \ell}=(-1)^{L} \sum_{a b}\left\langle a^{\prime} b^{\prime}|K(\gamma)| a b\right\rangle_{L \ell}\left\langle a b\left|K\left(\gamma^{\prime}\right)\right| a^{\prime \prime} b^{\prime \prime}\right\rangle_{L \ell}
\end{gathered}
$$




\section{3. МЕТОД}

Поставим задачу следующим образом: в случае взаимодействий (7) доказать в классе $\mathcal{B}^{\varepsilon}$ критерий сушествования и сформулировать метод построения всех решений уравнения Шредингера (10) типа

$$
\begin{gathered}
\Psi^{\varepsilon}\left(\mathbf{r}_{i} ; p^{2}\right)=r^{-2} Z_{p}(z) F^{\varepsilon}\left(\Omega_{i} ; p^{2}\right), \quad z \equiv r \sqrt{E} \\
F^{\varepsilon}\left(\Omega_{i} ; p^{2}\right)=\sum_{L=s}^{t} \sum_{a b}{ }^{i} B_{L a b}^{\ell} Y_{L a b}^{\ell m}\left(\Omega_{i}\right)
\end{gathered}
$$

где $s$ - минимально возможное при данном $\varepsilon$ значение гипермомента, $t<\infty$, а все коэффициенты ${ }^{i} B_{L a b}^{\ell}$ зависят от значений константы $p^{2}$.

Исследуемый случай парных взаимодействий центробежного типа (7) является исключительным: только такие взаимодействия зависят от гиперрадиуса таким же образом, как и единственное действующее на переменные $\Omega_{i}$ слагаемое $r^{-2} \mathbf{L}^{2}\left(\Omega_{i}\right)$ свободного гамильтониана (6). Поэтому после умножения уравнения Шредингера (10), записанного в представлении $\left\langle r, \Omega_{i}\right|$, на $r^{2}$ получается уравнение

$$
\left(r^{-3} \partial_{r}\left(r^{5} \partial_{r}\right)+r^{2} E\right) \Psi^{\varepsilon}\left(\mathbf{r}_{i}\right)=\left(\mathbf{L}^{2}\left(\Omega_{i}\right)+\sum_{k=1}^{3} I_{k}^{d} c_{k} \sec ^{2} \varphi_{k}\right) \Psi^{\varepsilon}\left(\mathbf{r}_{i}\right)
$$

в левой части которого все операторы действуют только на один аргумент $r$, а в правой части - на все остальные переменные $\Omega_{i}$. Дифференциальные уравнения, обладающие таким свойством, могут иметь факторизованные решения, которые строятся методом разделения переменных [10]. Применим этот метод к уравнению (21): подстановкой (19) сведем его к двум уравнениям, связанным посредством неизвестной пока постоянной $p^{2}$ разделения переменных $r$ и $\Omega_{i}$. Первое уравнение - хорошо изученное при всех комплексных $z$ и $p$ уравнение Бесселя [11] с пока не определенным $p$ :

$$
\left(z^{2} \partial_{z}^{2}+z \partial_{z}+z^{2}-p^{2}\right) Z_{p}(z)=0
$$

а второе уравнение можно интерпретировать как трехчастичное уравнение Шредингера на $\mathcal{S}^{5}$ с параметром $p^{2}-4$ в качестве энергии:

$$
\left(\mathbf{L}^{2}\left(\Omega_{i}\right)+\sum_{k=1}^{3} I_{k}^{d} c_{k} \sec ^{2} \varphi_{k}-\left(p^{2}-4\right)\right) F^{\varepsilon}\left(\Omega_{i} ; p^{2}\right)=0
$$

Поставленная задача частично решена: из уравнения Шредингера (10) определена функциональная зависимость его искомого решения (19) от $r$.

Аналитическое исследование всех решений уравнения (23) представляется очень сложной задачей по трем причинам: в этом уравнении содержится свободный параметр $p^{2}$, число независимых переменных $\hat{x}_{i}, \hat{y}_{i}, \varphi_{i}$, равное пяти, достаточно велико, более того, зависимость (9) функций $\cos ^{2} \varphi_{k}$ c $k \neq i$ от аргументов $\Omega_{i}$ не простая. Все переменные можно разделить, применив к обсуждаемому уравнению (23) метод гипергармоник. 
Подставим ряд (20) с пока не определенным $t$ в это уравнение. Полученное уравнение спроецируем на гиперсферический базис (13). Таким образом выведем, вообще говоря, бесконечную однородную систему линейных уравнений для неизвестных коэффициентов ${ }^{i} B_{L a b}^{\ell}$. Потенциальная часть матрицы этой системы содержит интегралы

$$
c_{k} \int_{\mathcal{S}^{5}} d \Omega_{i}\left(Y_{L a b}^{\ell m}\left(\Omega_{i}\right)\right)^{*} I_{k}^{d} \sec ^{2} \varphi_{k} Y_{L^{\prime} a^{\prime} b^{\prime}}^{\ell m}\left(\Omega_{i}\right), \quad k=1,2,3,
$$

которые с помощью формул (15) и известного представления [11] полинома $P_{n}^{(a+1 / 2, b+1 / 2)}(q)$ переменной $q \equiv \cos 2 \varphi_{i}$ в виде конечного ряда по целым степеням $(1 \pm q)$ сведем к конечным суммам табличных интегралов [12]. В итоге каждый исходньй интеграл представится конечной суммой, содержащей коэффициенты Рейнала-Реваи и гипергеометрические ряды ${ }_{3} F_{2}$ от комбинаций индексов $L, a, b, a^{\prime}$ и $b^{\prime}$. Из-за столь сложной зависимости потенциальных матричных элементов от пяти индексов аналитическое исследование системы уравнений для коэффициентов ${ }^{i} B_{L a b}$ представляется невозможным.

Первопричина того, что методом гипергармоник не удалось свести уравнение (23) к более простой задаче, такова: это уравнение, как и исходное уравнение Шредингера (10), содержит сумму трех взаимодействий и допускает запись только в одном координатном представлении, которое всегда оказывается несобственным для двух парных взаимодействий, что и приводит к сложным потенциальным матрицам. В отличие от уравнения Шредингера (10) каждое уравнение фаддеевской системы (12) содержит только одно взаимодействие и по определению записывается в собственном для этого взаимодействия координатном представлении. Поэтому уравнения Фаддеева более удобны для построения точных решений задачи трех частищ с любыми парными взаимодействиями, чем отвечающее им уравнение Шредингера. Приведенные ниже построения еще раз подтверждают этот известный факт [2].

Чтобы продолжить построение функций (19), необходимо определить постоянную $p$, индекс $t$ и коэффициенты ${ }^{i} B_{L a b}^{\ell}$ ряда (20). Для этого перейдем к уравнениям Фаддеева (12). Так как в искомой функции (19) переменные $r$ и $\Omega_{i}$ разделены, то ее фаддеевские компоненты имеют вид

$$
\Psi_{i}^{\varepsilon}\left(\mathbf{r}_{i} ; p^{2}\right)=r^{-2} Z_{p}(z) F_{i}^{\varepsilon}\left(\Omega_{i} ; p^{2}\right), \quad i=1,2,3,
$$

а функция $F^{\varepsilon}$ представляется суммой своих фаддеевских компонент $F_{k}^{\varepsilon}$ :

$$
F^{\varepsilon}\left(\Omega_{i} ; p^{2}\right)=\sum_{k=1}^{3} F_{k}^{\varepsilon}\left(\Omega_{k}\left(\Omega_{i} ; \gamma_{k i}\right) ; p^{2}\right)
$$

Используя представления (24) и (25) и тот факт, что $Z_{p}$ - решение уравнения (22), выводим из исходных уравнений Фаддеева $(12)$ в $\mathcal{R}^{6}$ уравнения Фаддеева на $\mathcal{S}^{5}$

$$
\cos ^{2} \varphi_{i}\left(\mathbf{L}^{2}\left(\Omega_{i}\right)+4-p^{2}\right) F_{i}^{\varepsilon}\left(\Omega_{i} ; p^{2}\right)=-c_{i} I_{i}^{d} \sum_{k=1}^{3} F_{k}^{\varepsilon}\left(\Omega_{k} ; p^{2}\right), \quad i=1,2,3
$$

5 Теоретическая и математическая физика, т. 128, № 2, 2001 г. 
Для углового анализа этой системы представим искомые функции $F_{i}^{\varepsilon}$ рядами по их собственнылм гиперсферическим базисам с пока неизвестными постоянными $t$ и $B_{L a b}^{i \ell}$ :

$$
F_{i}^{\varepsilon}\left(\Omega_{i} ; p^{2}\right)=\sum_{L=s}^{t} \sum_{a b} B_{L a b}^{i \ell} Y_{L a b}^{\ell m}\left(\Omega_{i}\right), \quad i=1,2,3
$$

Выведем два вспомогательных соотношения. Подставим ряды (20) и (27) в равенство (25). Затем, применяя правило (15), получим первое соотношение - связь между коэффициентами разложений функции $F^{\varepsilon}\left(\mathbf{r}_{i} ; p^{2}\right)$ и ее компонент $F_{k}^{\varepsilon}\left(\mathbf{r}_{k} ; p^{2}\right)$ :

$$
{ }^{i} B_{L a b}^{\ell}=B_{L a b}^{i \ell}+\sum_{k \neq i} \sum_{a^{\prime} b^{\prime}}\left\langle a b\left|K\left(\gamma_{k i}\right)\right| a^{\prime} b^{\prime}\right\rangle_{L \ell} B_{L a^{\prime} b^{\prime}}^{k \ell}
$$

Для вывода второго соотношения - разложения по гипергармоникам произведения $\cos ^{2} \varphi_{i} Y_{L a b}^{\ell m}\left(\Omega_{i}\right)$ - используем представление (13) и положим $q \equiv \cos 2 \varphi_{i}$ и $\cos ^{2} \varphi_{i}=(1+q) / 2$. Затем по известной формуле [11] представим функцию $q P_{n}^{(a+1 / 2, b+1 / 2)}(q)$ с индексом $n=(L-a-b) / 2 \neq 0$ линейной комбинацией двух полиномов $P_{n \pm 1}^{(a+1 / 2, b+1 / 2)}(q)$, а в оставшемся случае $n=0$ - произведением числового множителя на полином $P_{1}^{(a+1 / 2, b+1 / 2)}(q)$. Учтем также, что по определению индекса $s$ при данном $\varepsilon$ не сушествует гипергармоник с индексами гипермомента $L<s$. В итоге получим искомое и ключевое для дальнейших построений представление

$$
\cos ^{2} \varphi_{i} Y_{L a b}^{\ell m}\left(\Omega_{i}\right)=\sum_{L^{\prime}=L_{-}(L)}^{L+2} C_{L^{\prime} L}^{a b} Y_{L^{\prime} a b}^{\ell m}\left(\Omega_{i}\right),
$$

где $L_{-}(L) \equiv L-2\left(1-\delta_{L s}\right)\left(1-\delta_{L, a+b}\right), L=a+b+2 n$ и

$$
\begin{gathered}
C_{L L}^{a b}=\frac{1}{2}\left(1+\frac{(b-a)(a+b+1)}{(L+1)(L+3)}\right), \quad C_{L L^{\prime}}^{a b}=C_{L^{\prime} L}^{a b}, \\
C_{L, L-2}^{a b}=\frac{1}{4}\left(\left(1-\frac{a+b}{L}\right)\left(1+\frac{a+b}{L+2}\right)\right)^{\frac{1}{2}}\left(1-\left(\frac{a-b}{L+1}\right)^{2}\right)^{\frac{1}{2}} .
\end{gathered}
$$

Оно означает, что оператор умножения на функцию $\cos ^{2} \varphi_{i}$ отображает гипергармонику $Y_{L a b}^{\ell m}\left(\Omega_{i}\right)$ в линейную комбинацию гипергармоник с теми же самыми индексами $a$ и $b$ и индексами гипермомента $L, L+2$ в случае $L=s$ или $L=a+b$ и индексами $L, L \pm 2$ во всех остальных случаях.

Приступим к угловому анализу системы (26) в самом обшем случае трех центральных взаимодействий $(7)$, когда $I_{k}^{d}=I, k=1,2,3$. Каждое $(i=1,2,3)$ уравнение этой системы преобразуем следуюшим образом: подставим в него искомые ряды (27), затем применим формулы (14), (28) и (29), приведем подобные члены и получившееся соотношение спроецируем на собственные для исследуемого уравнения базисные гипергармоники $Y_{L a b}^{\ell m}\left(\Omega_{i}\right)$ с любыми допустимыми при данном $\varepsilon$ индексами $L, a$ и $b$. Упорядочим 
полученные линейные уравнения для искомых коэффициентов $B_{L a b}^{i \ell}$. Сначала скомпонуем $L$-блоки, составив каждьй $L$-блок из уравнений для коэффициентов $B_{L a b}^{i \ell}, i=1,2,3$, полученных проецированием всех трех уравнений (26)на гипергармоники с одним и тем же $L$, но всеми возможными при таком $L$ индексами $a$ и $b(L=a+b+2 n, n=0,1, \ldots)$. Число $N_{L \varepsilon}$ всех возможных пар таких индексов конечно. Внутри каждого $L$-блока, состоящего из $3 N_{L \varepsilon}$ уравнений, сначала запишем все $N_{L \varepsilon}$ уравнений для коэффициентов $B_{L a b}^{i \ell}$, порожденных проецированием на гипергармоники с одним и тем же индексом $L$ первого ( $i=1)$ уравнения системы $(26)$, затем второго $(i=2)$ и, наконец, третьего $(i=3)$. Упорядоченные таким образом $L$-блоки $(L=s, s+2, \ldots, t+2)$ запишем в порядке возрастания индекса $L$. Для сокрашения записи используем символы ${ }^{i} B_{L a b}^{\ell}$ линейных комбинаций (28) искомых коэффициентов $B_{L a b}^{i \ell}$. В итоге первые $L$-блоки с $L=s, s+2, \ldots, t-2$ дадут незамкнутую систему уравнений

$$
\sum_{L^{\prime}=L_{-}(L)}^{L_{+}(L)}\left(\left(L^{\prime}+2\right)^{2}-p^{2}\right) C_{L^{\prime} L^{a b}}^{a b} B_{L^{\prime} a b}^{i \ell}+c_{i}^{i} B_{L a b}^{\ell}=0
$$

где $L_{+}(L)=L+2$, а предел $L_{-}(L)$ - такой же, как в суммах (29). Предпоследний $L$-блок $(L=t)$ составят уравнения

$$
\left(t^{2}-p^{2}\right) C_{t-2, t}^{a b} B_{t-2, a b}^{i \ell}+\left((t+2)^{2}-p^{2}\right) C_{t t}^{a b} B_{t a b}^{i \ell}+c_{i}^{i} B_{t a b}^{\ell}=0,
$$

а последний $L$-блок $(L=t+2)$ - незацепляющиеся по индексам $i, a$ и $b$ уравнения

$$
\left((t+2)^{2}-p^{2}\right) C_{t, t+2}^{a b} B_{t a b}^{i \ell}=0 .
$$

Этим незацепляющимся уравнениям всегда удовлетворяют нулевые значения всех коэффициентов $B_{t a b}^{i \ell}$. В этом случае уравнения (32) совместны только при всех нулевых значениях $B_{t-2, a b}^{i \ell}$, так как все коэффициенты $C_{L^{\prime} L}^{a b}$ (см. формулы (30)) положительные, по той же причине система (31) имеет только тривиальное решение. Рассматриваемая система (33) имеет нетривиальные решения $\left(B_{t a b}^{i \ell} \neq 0\right.$ хотя бы при одной тройке индексов $i, a$ и $b$ ) тогда и только тогда, когда $p^{2}=(t+2)^{2}$. При таком подразумеваемом далее всюду соотношении последний $L$-блок $(L=t-2)$ системы $(31)$ не содержит неизвестных $B_{t a b}^{i \ell}$. Поэтому в этой системе далее полагаем $L_{+}(L)=L+2\left(1-\delta_{L, t-2}\right)$, а соотношения (32) переписываем в виде уравнений, определяюших неизвестные $B_{t a b}^{i \ell}$ через неизвестные $B_{t-2, a b}^{i \ell}$ :

$$
\begin{aligned}
c_{i}{ }^{i} B_{t a b}^{\ell} & =c_{i}\left(B_{t a b}^{i \ell}+\sum_{k \neq i} \sum_{a^{\prime} b^{\prime}}\left\langle a b\left|K\left(\gamma_{k i}\right)\right| a^{\prime} b^{\prime}\right\rangle_{t \ell} B_{t a^{\prime} b^{\prime}}^{k \ell}\right)= \\
& =4(t+1) C_{t, t-2}^{a b} B_{t-2, a b}^{i \ell} .
\end{aligned}
$$

Исследуем на совместность полученную совокупность систем (31) и (34). Возможны всего два случая: все коэффициенты $B_{t-2, a b}^{i \ell}$ нулевые или хотя бы один из них не равен нулю. 
Пусть все коэффициенты $B_{t-2, a b}^{i \ell}$ нулевые. Тогда система (34) становится однородной. Подставим в нее произвольные коэффициенты $B_{t a b}^{2 \ell}$ и $B_{t a b}^{3 \ell}$, а все коэффициенты $B_{t a b}^{1 \ell}$ выберем так, чтобы заведомо удовлетворялись все уравнения с индексом $i=1$ :

$$
B_{t a b}^{1 \ell}=-\sum_{k=2}^{3} \sum_{a^{\prime} b^{\prime}}\left\langle a b\left|K\left(\gamma_{k 1}\right)\right| a^{\prime} b^{\prime}\right\rangle_{t \ell} B_{t a^{\prime} b^{\prime}}^{k \ell}, \quad \forall B_{t a b}^{k \ell}, \quad \forall a, b .
$$

Затем, применяя формулы (4) и (16)-(18), сведем все полученные таким образом соотношения к тождествам типа $0=0$. Следовательно, однородная система, отвечающая системе (34), всегда имеет нетривиальные решения типа (35). Значит, по известной теореме линейной алгебры [13] матрица $\mathbf{M}^{t}$ этой системы всегда вырожденна $\left(\operatorname{det} \mathbf{M}^{t}=0\right)$.

Если все коэффициенты $B_{t-2, a b}^{i \ell}$ нулевые, то из системы (31) следует, что все коэффициенты $B_{L a b}^{i \ell}$ с $L<t-2$ также равны нулю. Поэтому трехкратные ряды (27) вырождаются в суммы по индексам $a$ и $b$ с неоднозначно определенными коэффициентами (35):

$$
F_{i}^{\varepsilon}\left(\Omega_{i} ; p^{2}\right)=\sum_{a b} B_{t a b}^{i \ell} Y_{t a b}^{\ell m}\left(\Omega_{i}\right), \quad i=1,2,3
$$

При любых таких коэффициентах в сумме (20) все коэффициенты (28) равны нулю, а сама сумма - тривиальная функция: $F^{\varepsilon} \equiv 0$. Следовательно, найденные нетривиальные решения (36) уравнений Фаддеева (26) - ложные решения [3].

Пусть теперь хотя бы один из коэффициентов $B_{t-2, a b}^{i \ell}$ не равен нулю. Тогда система (34) несовместна как неоднородная система с вырожденной матрицей $\mathbf{M}^{t}$.

Итак, в случае центральных взаимодействий (7) уравнение Шредингера (10) никогда не имеет нетривиальных решений типа (19), (20), а уравнения Фаддеева (12) имеют ложные решения, заданные формулами $(24),(35)$ и (36).

Исследуем теперь случай нецентральных взаимодействий: $I_{i}^{d} \neq I, i=1,2,3$, на $\mathcal{S}^{5}$.

Пусть все три взаимодействия $V_{k}$ - последовательные $(b=0,1, \ldots, d<\infty)$ конечные подсуммы разложения оператора $I$ по соответствующим сферическим базисам. Тогда оператор $I_{i}^{d}$ является единичным для всех гипергармоник $Y_{L a b}^{\ell m}\left(\Omega_{i}\right)$ с индексом $b \leqslant d$ и эквивалентен оператору умножения на нуль для всех остальных гипергармоник тех же аргументов.

Пусть в искомых рядах (20) и (27) максимальное значение $t$ гипермомента настолько мало, что в представлении $t=a+b+2 n$, где $n=0,1, \ldots$, все возможные при данном $\varepsilon$ и всех допустимых $n$ значения $b$ не превьшают $d$. Тогда на линейной оболочке гипергармоник $Y_{L a b}^{\ell m}\left(\Omega_{i}\right)$ с $L=s, \ldots, t$ оператор $I_{i}^{d}$ эквивалентен единичному оператору. Поэтому в результате проецирования уравнений (26) на все такие гипергармоники получатся системы (31) и (32), в которых индексы $a^{\prime}, b^{\prime}, a$ и $b$ будут принимать, как и в выше рассмотренном случае $(d=\infty)$, все допустимые определением гипергармоник (13) значения. Индексы $a$ и $b$ в проекциях (33) уравнений $(26)$ на гипергармоники $Y_{t+2, a b}^{\ell m}$ окажутся ограниченными условием $b \leqslant d$. Оно никак не повлияет ни на анализ системы $(31)-(33)$ описанным выше способом, ни на окончательные выводы об отсутствии в рассмотренном случае нетривиальных решений (19), (20) уравнения Шредингера (21). 
Теперь предположим, что в искомых рядах (20) и (27) значение $t$ настолько велико, что хотя бы для одной пары слагаемых $a$ и $2 n$ в сумме $t=a+b+2 n$ индекс $b$ может превышать $d$. Тогда матричные элементы

$$
\int_{\mathcal{S}^{5}} d \Omega_{i}\left(Y_{t a b}^{\ell m}\left(\Omega_{i}\right)\right)^{*} I_{i}^{d} Y_{t a b^{\prime}}^{\ell m}\left(\Omega_{i}\right)
$$

равны единише при $b^{\prime}=b \leqslant d$ и нулю при $b^{\prime} \neq b$ или $b^{\prime}=b>d$. Поэтому проецированием уравнений (26) на весь базис (13) получатся уравнения

$$
\left((L+2)^{2}-p^{2}\right) B_{L a b}^{i \ell}=0, \quad L=s, s+2, \ldots, t, \quad b>d
$$

и уравнения (31)-(33), в которых индексы $a^{\prime}, b^{\prime}, a$ и $b$ подчиняются дополнительным к перечисленным в формулах (13) условиям $b^{\prime}, b \leqslant d$. Удовлетворим эти уравнения, положив $B_{L a b}^{i \ell}=0$ при всех $L \leqslant t$ и $b>d$. Из равенств (33) с $b<d$ опять получаем $p^{2}=$ $(t+2)^{2}$. Теперь в рядах $(20),(27)$ и суммах $(28)$ индексы $b^{\prime}$ и $b$ не превьшают $d$, а значения индексов $a^{\prime}$ и $a$ также оказываются ограниченными условиями треугольника.

Если, несмотря на дополнительные ограничения на индексы, матрица $\mathbf{M}^{t}$ системы (34) вырожденна, то, как и в случае центральных взаимодействий, имеются только ложные решения (35), (36) уравнений Фаддеева (26). Если же благодаря этим ограничениям $\operatorname{det} \mathbf{M}^{t} \neq 0$, то возможны два случая: матрица $\mathbf{A}^{t}$ системы (31) вырожденна или невырожденна. Пусть $\operatorname{det} \mathbf{A}^{t} \neq 0$, тогда эта система имеет лиш тривиальное решение, и поэтому такое же решение имеет система (34). Если же $\operatorname{det} \mathbf{A}^{t}=0$, то система (31), a значит, и система (34) имеют нетривиальные решения.

Итак, если в случае нецентральных взаимодействий, заданных формулами (7), в которых $b=0,1, \ldots, d<\infty$, выполняются условия

$$
p^{2}=(t+2)^{2}, \quad t>d, \quad \operatorname{det} \mathbf{A}^{t}=0, \quad \operatorname{det} \mathbf{M}^{t} \neq 0
$$

а коэффициенты $B_{L a b}^{i \ell}$ удовлетворяют системам $(31)$ и $(34)$, то функция $\Psi^{\varepsilon}$, заданная формулами (19), (20) и (28), - точное решение уравнения Шредингера (21).

Докажем, что эти же условия являются и необходимыми. Пусть функция $\Psi^{\varepsilon}$ типа (19), (20) удовлетворяет уравнению Шредингера (21). Дословно повторим вывод соотношения $p^{2}=(t+2)^{2}$ и систем $(31),(34)$. Теперь коэффициенты $B_{L a b}^{i \ell}$ удовлетворяют этим системам по определению. Так как однородная система (31) имеет решение, то ее матрица $\mathbf{A}^{t}$ вырожденна. Неоднородная система (34) тоже имеет решение. Следовательно, $\operatorname{det} \mathbf{M}^{t} \neq 0$, что, как было показано, возможно только при $t>d$.

Исследуем доказанный критерий сушествования (37) точных решений. Элементы матрицы $\mathbf{A}^{t}$ и $\mathbf{M}^{t}$ систем (31) и (34) зависят от параметров $c_{1}, c_{2}, c_{3}$ взаимодействий и от кинематических углов - функций (3) двух отношений масс частиц, например $m_{1} / m_{3}$ и $m_{2} / m_{3}$. Поэтому условие $\operatorname{det} \mathbf{M}^{t} \neq 0$ может выполняться при любых ненулевых параметрах $c_{1}, c_{2}$ и $c_{3}$, но не при всех отношениях масс частиц, а равенство $\operatorname{det} \mathbf{A}^{t}=0$ может быть справедливым только при определенных отношениях масс частиц и значениях этих параметров. 
Пусть $N_{L \varepsilon}^{d}$ - число гипергармоник $Y_{L a b}^{\ell m}$ с индексами $b \leqslant d$ и любыми допустимыми при таких $b$ индексами $a$. Так как каждый $L$-блок системы $(31)$ содержит $N_{L \varepsilon}^{d}$ уравнений, то матрица $\mathbf{A}^{t}$ имеет размерность

$$
N=3 \sum_{L=s}^{t-2} N_{L \varepsilon}^{d}
$$

и является ленточной матрицей с шириной ленты $3 N_{t-2, \varepsilon}^{d}$. Ее детерминант удобно представить полиномом степени $N$ по целым степеням аргумента $q$ - одного из параметров $c_{1}$, $c_{2}, c_{3}$ взаимодействий (7), с коэффициентами $C_{n}$, зависящими от двух других параметров и двух отношений масс частиц. Массу одной из частиц можно принять за единицу масс. При нашем выборе $q=c_{2}$ и $m_{3}=1$ получается характеристическое уравнение

$$
\operatorname{det} \mathbf{A}^{t}=\sum_{n=0}^{N} C_{n}\left(c_{1}, c_{3}, m_{1}, m_{2}\right) c_{2}^{n}=0
$$

а анализ условия $\operatorname{det} \mathbf{A}^{t}=0$ сводится к исследованию всех его действительных нулей $c_{2}\left(c_{1} ; c_{3}, m_{1}, m_{2}\right)$ как функций аргумента $c_{1}$ и параметров $c_{3}, m_{1}, m_{2}$, причем параметры $m_{1}$ и $m_{2}$ берутся лишь такие, при которых $\operatorname{det} \mathbf{M}^{t}\left(m_{1}, m_{2}\right) \neq 0$.

Итак, случай взаимодействий (7) с индексом суммирования $b=1,2, \ldots, d<\infty$ полностью рассмотрен. Все остальные случаи, когда индекс $b$ пробегает произвольную, но конечную последовательность $(b \leqslant d<\infty)$, можно исследовать тем же способом и показать, что независимо от выбора такой последовательности сформулированный выше критерий остается в силе.

До сих пор три частицы считались разными. Для полноты стоит рассмотреть и два оставшихся случая тождественных частиц.

Пусть две из трех частиц, для определенности $p_{2}$ и $p_{3}$, тождественные. Тогда $m_{2}=$ $m_{3}, c_{2}=c_{3}$ и согласно определениям (3) $\gamma_{12}=\gamma_{31}$, а на решение $\Psi^{\varepsilon}$ уравнения Шредингера (10) и его три фаддеевские компоненты $\Psi_{k}^{\varepsilon}$ накладьваются известные условия перестановочной симметрии [2]:

$$
\Psi^{\varepsilon}= \pm P_{23} \Psi^{\varepsilon} ; \quad \Psi_{1}^{\varepsilon}= \pm P_{23} \Psi_{1}^{\varepsilon}, \quad \Psi_{2}^{\varepsilon}= \pm P_{23} \Psi_{3}^{\varepsilon}, \quad \Psi^{\varepsilon}=\Psi_{1}^{\varepsilon}+\left(I \pm P_{23}\right) \Psi_{2}^{\varepsilon}
$$

Здесь и далее, где не оговорено, берется знак плюс или минус, если $p_{2}$ и $p_{3}$ бозоны или соответственно фермионы. Свойства перестановочной симметрии (14) гипергармоник и условия (39) порождают дополнительные ограничения на коэффициенты $B_{L a b}^{i \ell}$ рядов фаддеевских компонент (27) и комбинаций $(28): B_{L a b}^{1 \ell}=0$ при всех $L, a$ и нечетном (четном) $b$, если $p_{1}$ и $p_{2}-$ бозоны (фермионы) и в любом случае $B_{L a b}^{2 \ell}=B_{L a b}^{3 \ell}$ при всех $L, a$ и $b$. Из-за таких условий системы (31) и (34) упрощаются: в них индекс $i$ принимает теперь не три, а два значения $(i=1,2)$, при $i=1$ индексы $b^{\prime}$ и $b$ только четные (нечетные), 
если $p_{2}, p_{3}$ - бозоны (фермионы), а комбинации (28) вычисляются по формулам

$$
\begin{aligned}
{ }^{1} B_{1 L a b}^{\ell}= & B_{L a b}^{1 \ell}+\left((-1)^{b} \pm 1\right) \sum_{a^{\prime} b^{\prime}}(-1)^{b^{\prime}}\left\langle a b\left|K\left(\gamma_{12}\right)\right| a^{\prime} b^{\prime}\right\rangle_{L \ell \ell} B_{L a^{\prime} b^{\prime}}^{2 \ell}, \\
{ }^{2} B_{L a b}^{\ell}= & \sum_{a^{\prime} b^{\prime}}\left\langle a b\left|K\left(\gamma_{12}\right)\right| a^{\prime} b^{\prime}\right\rangle_{L \ell} B_{L a^{\prime} b^{\prime}}^{1 \ell}+ \\
& +B_{L a b}^{2 \ell} \pm(-1)^{b} \sum_{a^{\prime} b^{\prime}}\left\langle a b\left|K\left(\gamma_{23}\right)\right| a^{\prime} b^{\prime}\right\rangle_{L \ell^{2}} B_{L a^{\prime} b^{\prime}}^{2 \ell}
\end{aligned}
$$

Пусть теперь все три частицы тождественные. Тогда при любых $i, k=1,2,3$ верны равенства $m_{i}=m_{k}, c_{i}=c_{k},\left|\gamma_{k i}\right|=\pi / 3$. Условия симметрии [2]

$$
\Psi^{\varepsilon}= \pm P_{k i} \Psi^{\varepsilon} ; \quad \Psi_{i}^{\varepsilon}= \pm P_{k i} \Psi_{k}^{\varepsilon}, \quad \Psi^{\varepsilon}=\left(I \pm P_{12} \pm P_{23}\right) \Psi_{i}^{\varepsilon}, \quad i \neq k=1,2,3,
$$

будут выполняться для искомой функшии (19), если в рядах (20), (27) и суммах (28) положить $B_{L a b}^{1 \ell}=B_{L a b}^{2}=B_{L a b}^{3 \ell}$ при всех $L, a$ и $b$, а индексы $b^{\prime}$ и $b$ брать четные или нечетные, если три частицы - бозоны или фермионы соответственно. Тогда в системах (31) и (34) индекс $i$ будет принимать лишь одно значение $(i=1)$, индексы $b^{\prime}$ и $b$ подчинятся тем же условиям четности, а формулы (28) примут совсем простой вид:

$$
{ }^{1} B_{L a b}^{\ell}=\sum_{a^{\prime} b^{\prime}}\left(\delta_{a a^{\prime}} \delta_{b b^{\prime}}+2\left\langle a b\left|K\left(\frac{\pi}{3}\right)\right| a^{\prime} b^{\prime}\right\rangle_{L \ell}\right) B_{L^{\prime} a^{\prime} b^{\prime}}^{1 \ell}
$$

Учет описанных ограничений на коэффициенты $B_{L a b}^{i \ell}$ и индексы не вносит ничего сушественного в анализ систем (31) и (34), а значит, и в его окончательные выводы: уравнение Шредингера (21) не имеет факторизованных точных решений (19), (20) с перестановочной симметрией (39) или (41) в случае центральных взаимодействий и обладает такими в случае нецентральных взаимодействий при условиях (37).

\section{4. ПРИМЕРЫ}

Для пояснения предложенного метода проанализируем самый простой случай. Пусть все взаимодействия $S$-волновые, а полный угловой момент равен нулю.

Так как $d=0$ и $\ell=0$, то полный угловой базис образуют гипергармоники $Y_{L 00}^{00}$, индексы $s, a^{\prime}, b^{\prime}, a$ и $b$ могут принимать всюду только нулевые значения, а $L$ и $t$ - всегда четные числа: $L=2(n-1), t=2(m-1)$, где $m$ и $n$ натуральные. Для всех искомых и известных коэффициентов с такими индексами удобно ввести сокрашенные обозначения:

$$
\begin{gathered}
B_{n}^{i} \equiv B_{L a b}^{i \ell}, \quad{ }^{i} B_{n} \equiv{ }^{i} B_{L a b}, \\
K_{n}\left(\gamma_{k i}\right) \equiv\left\langle 00\left|K\left(\gamma_{k i}\right)\right| 00\right\rangle_{L 0}=\frac{\sin 2 n \gamma_{k i}}{n \sin 2 \gamma_{k i}} .
\end{gathered}
$$

Тогда искомые функции (19) и (24) примут вид

$$
\begin{aligned}
& \Psi^{\varepsilon}\left(r, \varphi_{i} ; 4 m^{2}\right)=(2 \pi)^{-\frac{3}{2}} r^{-2} Z_{2 m}(z) \sum_{n=1}^{m} B_{n} \frac{\sin 2 n \varphi_{i}}{\sin 2 \varphi_{i}} \\
& \Psi_{i}^{\varepsilon}\left(r, \varphi_{i} ; 4 m^{2}\right)=(2 \pi)^{-\frac{3}{2}} r^{-2} Z_{2 m}(z) \sum_{n=0}^{m} B_{n}^{i} \frac{\sin 2 n \varphi_{i}}{\sin 2 \varphi_{i}} .
\end{aligned}
$$


Коэффиициенты (30) с $a=0$ и $b=0$ не зависят от $L, L^{\prime}: C_{L L}^{00}=1 / 2$, а $C_{L, L \pm 2}^{00}=1 / 4$. Поэтому последовательными подстановками (43) и

$$
B_{n}^{i}=\frac{X_{n}^{i}}{n^{2}-m^{2}}, \quad n=1,2, \ldots, m-1 ; \quad X_{m}^{i}=B_{m}^{i}
$$

в определения (28), (40), (42) и системы (31) и (34), соответствуюшие рассматриваемой перестановочной симметрии, получаем более удобные для анализа систему матричных уравнений

$$
\begin{aligned}
\mathbf{D}^{1} \mathbf{X}_{1}+\mathbf{X}_{2} & =0 \\
\mathbf{X}_{n-1}+\mathbf{D}^{n} \mathbf{X}_{n}+\mathbf{X}_{n+1} & =0, \quad n=2,3, \ldots, m-2, \\
\mathbf{X}_{m-2}+\mathbf{D}^{m-1} \mathbf{X}_{m-1} & =0
\end{aligned}
$$

и соответственно одно матричное уравнение

$$
\mathbf{M}^{t} \mathbf{X}_{m}=-\frac{\mathbf{X}_{m-1}}{4}
$$

Систему (47) можно представить и одним матричным уравнением

$$
\mathbf{A}^{t} \mathbf{X}=0, \quad \mathbf{X}=\left(\mathbf{X}_{1}, \mathbf{X}_{2}, \ldots, \mathbf{X}_{m-1}\right)^{T}
$$

где $\mathbf{A}^{t}$ - блочно-трех диагональная матрица. Блоки ее главной диагонали-матрицы $\mathbf{D}^{n}$, а все блоки нижней и верхней диагоналей - единичные матришы.

Если все частицы разные, то в полученных уравнениях (47) и (48) все столбцы новых неизвестных коэффициентов $X_{n}^{i}$ и матрицы трехмерные:

$$
\begin{gathered}
\mathbf{X}_{n} \equiv\left(X_{n}^{1}, X_{n}^{2}, X_{n}^{3}\right)^{T}, \quad n=1,2, \ldots, m, \\
D_{i i}^{n}=2+\frac{c_{i}}{n^{2}-m^{2}}, \quad D_{i k}^{n}=\left(D_{i i}^{n}-2\right) K_{n}\left(\gamma_{k i}\right) ; \\
M_{i i}^{t}=c_{i}, \quad M_{i k}^{t}=c_{i} K_{m}\left(\gamma_{k i}\right), \quad k \neq i=1,2,3 .
\end{gathered}
$$

Если частицы $p_{2}$ и $p_{3}-$ тождественные бозоны, то из условия симметрии $B_{L a b}^{2 \ell}=B_{L a b}^{3 \ell}$ следует, что $X_{n}^{2}=X_{n}^{3}$ при всех $n$, поэтому все столбцы и матрицы становятся двухмерными:

$$
\begin{gathered}
\mathbf{X}_{n} \equiv\left(X_{n}^{1}, X_{n}^{2}\right)^{T}, \quad n=1,2, \ldots, m, \\
D_{i i}^{n}=2+\frac{c_{i}}{n^{2}-m^{2}}\left(1+\delta_{i 2} K_{n}\left(\gamma_{23}\right)\right), \quad D_{i k}^{n}=\frac{c_{i}}{n^{2}-m^{2}}\left(1+\delta_{k 2}\right) K_{n}\left(\gamma_{12}\right) ; \\
M_{i i}^{t}=c_{i}\left(1+\delta_{i 2} K_{m}\left(\gamma_{23}\right)\right), \quad M_{i k}^{t}=c_{i}\left(1+\delta_{k 2} K_{m}\left(\gamma_{k i}\right)\right), \quad k \neq i=1,2 .
\end{gathered}
$$

Если $p_{1}, p_{2}, p_{3}$ - тождественные бозоны, то из-за условий симметрии $\left(X_{n}^{i}=X_{n}^{k}\right.$ при любых $i, k, n)$ все столбцы и матрицы уравнений (47) и (48) являются одномерными:

$$
\mathbf{X}_{n} \equiv X_{n}^{1}, \quad \mathbf{D}^{n}=2+\frac{c_{2}}{n^{2}-m^{2}}\left(1+2 K_{n}\left(\frac{\pi}{3}\right)\right) ; \quad \mathbf{M}^{t}=c_{2}\left(1+2 K_{m}\left(\frac{\pi}{3}\right)\right) .
$$


Опишем наиболее экономньй способ решения системы (47), основанньй на известном методе прямой и обратной подстановок и удобный для операторного накопления [14]. Сначала все столбцы $\mathbf{X}_{n}$, начиная со второго, последовательно выражаем через первый столбец:

$$
\mathbf{X}_{2}=-\mathbf{D}^{1} \mathbf{X}_{1}, \quad \mathbf{X}_{3}=\left(\mathbf{D}^{2} \mathbf{D}^{1}-\mathbf{I}\right) \mathbf{X}_{1}, \ldots
$$

Таким образом исходную систему (47) или (49) сколь угодно большой, но конечной размерности сводим к уравнению

$$
\mathbf{G}^{t} \mathbf{X}_{1}=0
$$

По построению размерность его матришы $\mathbf{G}^{t}$ не превышает трех, а характеристическое уравнение (38) эквивалентно уравнению

$$
\operatorname{det} \mathbf{G}^{t}=0
$$

После того как найден корень $c_{2}$ этого уравнения и соответствуюшее ему решение $\mathbf{X}_{1}$ уравнения (54), все остальные столбцы $\mathbf{X}_{n}, n=2, \ldots, m-1$, последовательно вычисляем по формулам (53).

Как известно [15], матрицы $\mathbf{M}^{t}$ с элементами (50) или (51) вырожденны при $t=0,2$ и невырожденны при $t=4,6, \ldots$. Следовательно, если $t=4,6, \ldots$, а параметры $c_{1}, c_{2}, c_{3}$ взаимодействий удовлетворяют уравнению (55), то функции (44) и (45) будут нетривиальными точными решениями соответствуюших уравнений (10) и (12). Как пример исследуем уравнение (55) в случае $t=4$, когда $m=t / 2+1=3$ и $\mathbf{G}^{4}=\mathbf{I}-\mathbf{D}^{2} \mathbf{D}^{1}$.

4.1. Три разные частицы. Характеристическое уравнение (55) является квадратным:

$$
\begin{gathered}
\operatorname{det} \mathbf{G}^{4}=A c_{2}^{2}+B c_{2}+C=0 \\
A \equiv 15+2\left(t_{1}^{2}-1\right) c_{1}+2\left(t_{3}^{2}-1\right) c_{3} \\
B \equiv 2\left(t_{1}^{2}-1\right) c_{1}^{2}+2\left(t_{3}^{2}-1\right) c_{3}^{2}-390- \\
\quad-2\left(16 t_{1}^{2}+5 t_{1}-36\right) c_{1}-2\left(16 t_{3}^{2}+5 t_{3}-36\right) c_{3}+ \\
\quad+\frac{2}{3}\left(4\left(t_{1}^{2}+t_{2}^{2}+t_{3}^{2}\right)+t_{1}+t_{2}+t_{3}-12\right) c_{1} c_{3}- \\
\quad-\frac{2}{3}\left(\cos 2\left(\gamma_{12}-\gamma_{31}\right)+\cos 2\left(\gamma_{12}-\gamma_{23}\right)+\cos 2\left(\gamma_{31}-\gamma_{23}\right)\right) c_{1} c_{3} \\
C \equiv 15\left(c_{1}^{2}+c_{3}^{2}\right)+2\left(\left(t_{2}^{2}-1\right)\left(c_{1}+c_{3}\right)-16 t_{2}^{2}-5 t_{2}+36\right) c_{1} c_{3}- \\
\quad-390\left(c_{1}+c_{3}\right)+1800, \\
t_{1} \equiv \cos 2 \gamma_{12}, \quad t_{2} \equiv \cos 2 \gamma_{31}, \quad t_{3} \equiv \cos 2 \gamma_{23},
\end{gathered}
$$

и поэтому может иметь не более двух действительных решений: $c_{2}=c_{2}^{+}$и $c_{2}=c_{2}^{-}$. Их исследование начнем с предельных случаев (5). 
При $m_{1}=0$ в исследуемом уравнении (56) $t_{1}, t_{2}=-1, t_{3}=1$, поэтому $A=15$ и при любом $c_{3}$ дискриминант $D=B^{2}-4 A C>0$. Значит, имеются два разных $\left(c_{2}^{+} \neq c_{2}^{-}\right)$ непрерывных по $c_{1}$ и не зависящих от $m_{2}$ решения:

$$
c_{2}^{ \pm}\left(c_{1} ; c_{3}, 0, m_{2}\right)=-\frac{5}{3} c_{1}-c_{3}+13 \pm \frac{1}{3}\left(16 c_{1}^{2}-156 c_{1}+441\right)^{\frac{1}{2}}
$$

Если $m_{1}=\infty$ и $m_{2}=m_{3}$, то при любом $c_{3}$ в точке $c_{1}=c_{1}^{s} \equiv 15 / 2$ коэффициент $A=$ $15-2 c_{1}$ обрашается в нуль, но всегда $B \neq 0$ и $D>0$. Поэтому решение $c_{2}^{+}$терпит разрыв второго рода в точке $c_{1}^{s}$, а решение $c_{2}^{-}$непрерывно:

$$
c_{2}^{ \pm}\left(c_{1} ; c_{3}, m_{1}, m_{2}\right)=-\frac{B^{2}}{2 A}\left(1 \pm \operatorname{sign}\left(c_{1}^{s}-c_{1}\right)\left(1-4 \frac{A C}{B^{2}}\right)^{\frac{1}{2}}\right)
$$

Рассмотрим все оставшиеся случаи, когда $m_{1}, m_{2} \in(0, \infty)$. Теперь в отличие от случая $m_{1}=\infty$ положение нуля $c_{1}^{s}$ функции $A$ зависит от $c_{3}$,

$$
c_{1}=c_{1}^{s} \equiv \frac{15+2\left(t_{3}^{2}-1\right) c_{3}}{2\left(1-t_{1}^{2}\right)},
$$

но $B \neq 0$ и $D>0$ всегда, поэтому сушествуют два разных решения (57), причем решение $c_{2}^{+}$терпит разрыв в точке $c_{1}=c_{1}^{s}$, а решение $c_{2}^{-}-$всюду непрерывная функция.

Для наглядного представления всех характерных особенностей семейства решений $c_{2}^{ \pm}$ на рис. 1 в координатной плоскости $\left(c_{1}, c_{2}\right)$ изображены графики функций $c_{2}^{ \pm}$при одинаковых $m_{1}=2$ и $m_{2}=4$, но разных $c_{3}$, равных -2 и +2 . Перечислим главные особенности функций $c_{2}^{ \pm}$. График решения $c_{2}^{+}$всегда имеет горизонтальную, $c_{1}=c_{1}^{s}$, и вертикальную,

$$
c_{2}=c_{2}^{s} \equiv \frac{15+2\left(t_{2}^{2}-1\right) c_{3}}{2\left(1-t_{1}^{2}\right)},
$$

асимптоты, пересекающиеся в точке $\left(c_{1}^{s}, c_{2}^{s}\right)$, лежашей на прямой

$$
c_{2}=\frac{1-t_{2}^{2}}{1-t_{1}^{2}} c_{1}+\frac{15}{2}\left(t_{2}^{2}-t_{1}^{2}\right)
$$

При любых фиксированных $m_{1}, m_{2}$ и $c_{3}$ выполняются неравенства $c_{2}^{+}<c_{2}^{s}$, если $c_{1}<c_{1}^{s}$, и $c_{2}^{+}>c_{2}^{s}$, если $c_{1}>c_{1}^{s}$. Поэтому если $c_{3}$ возрастает или убывает, то точка $\left(c_{1}^{s}, c_{2}^{s}\right)$ смешается по этой прямой вниз или соответственно вверх; таким же образом при изменении $c_{3}$ смещаются и графики функций $c_{2}^{ \pm}$. Следовательно, при любых массах и параметре $c_{3}$ для любого $c_{1}$ найдутся два значения $c_{2}=c_{2}^{ \pm}$такие, что соответствующие им две функции (44) с индексом $m=3$ будут точными решениями уравнения Шредингера (21). В трехчастичных состояниях, описьваемых такими всюду регулярными волновыми функциями, ни при каких $E \geqslant 0, c_{1}, c_{3}$ и $c_{2}=c_{2}^{ \pm}$не происходит "падения" частиц ни в точку тройного $(r=0)$, ни в точки парных $\left(x_{k}=0, k=1,2,3\right)$ столкновений. Из определений коэффициентов (56) и формул (57) следует, что $c_{2}^{-} \rightarrow \infty$ при 


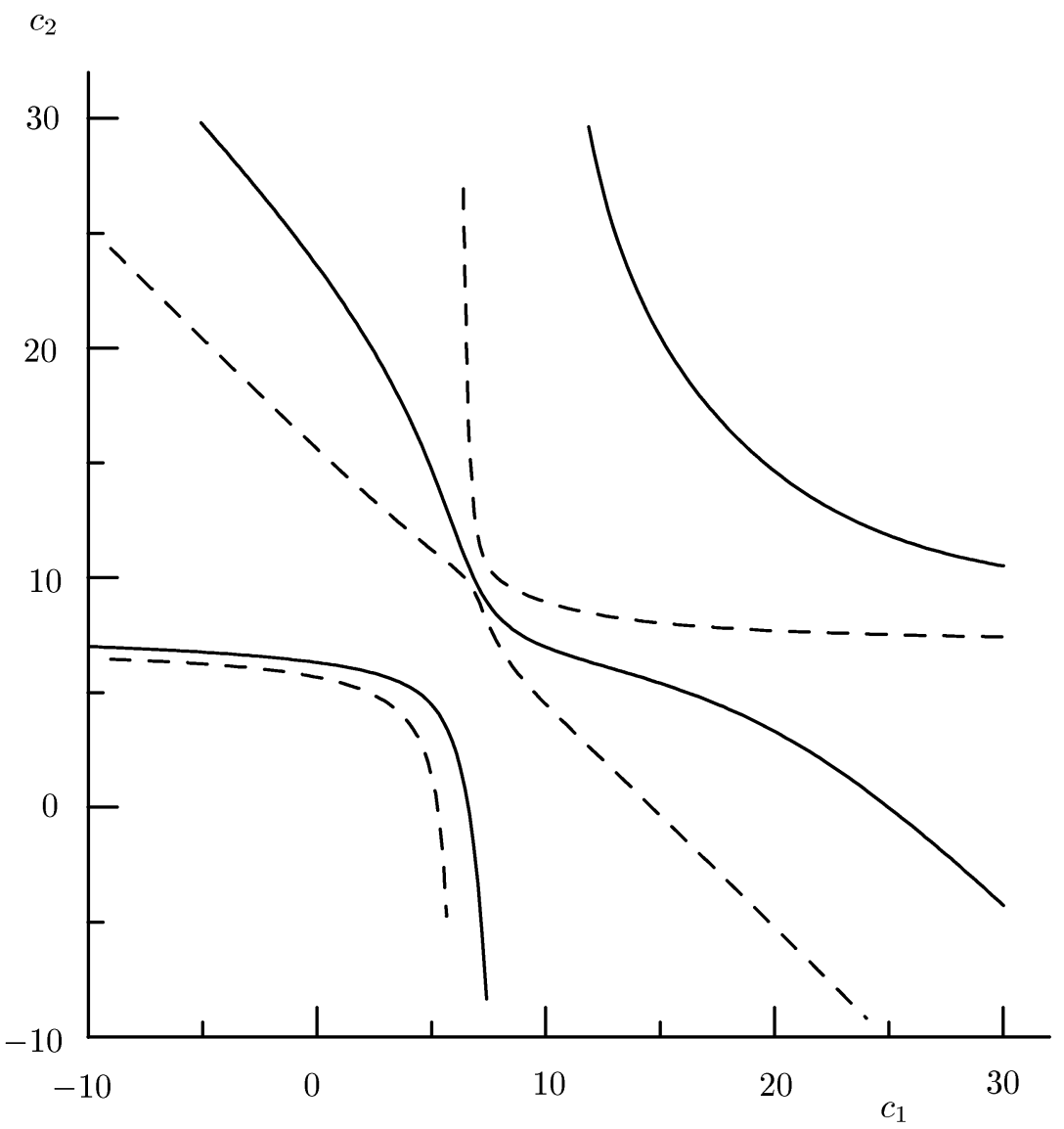

Рис. 1. Функции $c_{2}^{ \pm}\left(c_{1} ; c_{3}, m_{1}, m_{2}\right)$ при $m_{1}=2, m_{2}=4, c_{3}=-2$ (сплошные линии) и при $c_{3}=2$ (штриховые).

$c_{1} \rightarrow-\infty$ и любом $c_{3}$. Значит, даже если условие (1) при $c=c_{1}$ и $b=0$ не выполняется $\left(c_{1}<-1 / 4\right)$ и две частицы $p_{2}$ и $p_{3}$ в отсутствие третьей “падают друг на друга", то в трехчастичной системе $\left(p_{1}, p_{2}, p_{3}\right)$ их "падение" в состоянии с волновой функций (44) предотврашается отталкивающим взаимодействием между частицами $p_{1}$ и $p_{3}$. В этом смысле сильное притяжение $\left(c_{1} \rightarrow-\infty\right)$ в одной паре частиц компенсируется соответствующим $\left(c_{2}=c_{2}^{-} \rightarrow \infty\right)$ сильным отталкиванием в другой паре частищ, и асимптотическое поведение корня $c_{2}^{-}$характеристического уравнения $(56)$ интуитивно понятно. Поведение корня $c_{2}^{+}$при $c_{1} \rightarrow c_{1}^{s}-0$ выглядит необычно. В этом случае (см. рис. 1$)$ бесконечно сильное притяжение $\left(c_{2}=c_{2}^{+} \rightarrow-\infty\right)$ в паре $\left(p_{1}, p_{3}\right)$ компенсируется конечныцми $\left(c_{1}<c_{1}^{s}, c_{3}<\infty\right)$ отталкиваниями в других парах частиц.

4.2. Две частицы - тождественные бозоны. Теперь, в отличие от предыдушего случая, всегда $m_{2}=m_{3}$ и $c_{2}=c_{3}$. Поэтому уравнение (55) более простое:

$$
\operatorname{det} \mathbf{G}^{4}=A c_{2}^{2}+B c_{2}+C=0
$$




$$
\begin{gathered}
A \equiv 12 t_{1}^{2}, \quad B \equiv 4\left(\left(2 t_{1}^{2}-t_{1}+2\right) c_{1}-24 t_{1}^{2}-15\right) \\
C \equiv 3\left(c_{1}^{2}-6\right)\left(c_{1}-20\right), \quad t_{1} \equiv-\left(1+m_{1}\right)^{-1}
\end{gathered}
$$

а его действительные решения $c_{2}=c_{2}^{ \pm}\left(c_{1} ; m_{1}\right)$ зависят от одного аргумента $c_{1}$ и одного параметра $m_{1}$. Анализ всех таких решений начнем с предельных случаев (5).

При $m_{1}=0$ в уравнении (58) $t_{1}=1, A>0$ и $D=B^{2}-4 A C>0$ для любых $c_{1}$. Поэтому его решения разные и нелинейные по $c_{1}$ :

$$
c_{2}^{ \pm}\left(c_{1} ; 0\right)=-\frac{5}{6} c_{1}+\frac{13}{2} \pm \frac{1}{6}\left(16 c_{1}^{2}-156 c_{1}+441\right)^{\frac{1}{2}} .
$$

Если $m_{1}=\infty$, то $A=0$, а единственное решение терпит разрыв при $c_{1}=c_{1}^{s}=15 / 2$ :

$$
c_{2}\left(c_{1} ; \infty\right)=\frac{3}{4} \frac{\left(c_{1}-6\right)\left(c_{1}-20\right)}{15-2 c_{1}}
$$

В особом случае $m_{1}=3 / 5$ дискриминант $D$ - полный квадрат переменной $c_{1}$, поэтому оба решения - линейные функции аргумента $c_{1}$ :

$$
c_{2}^{+}\left(c_{1} ; \frac{3}{5}\right)=-\frac{6}{25} c_{1}+\frac{24}{5}, \quad c_{2}^{-}\left(c_{1} ; \frac{3}{5}\right)=-\frac{8}{3} c_{1}+16 .
$$

При любых $c_{1}$ и $m_{1} \neq 0,3 / 5, \infty$ дискриминант $D>0$, поэтому имеются два различных нелинейных по аргументу $c_{1}$ решения:

$$
\begin{aligned}
c_{2}^{ \pm}\left(c_{1} ; m_{1}\right)= & \frac{\left(t_{1}-2 t_{1}^{2}-2\right) c_{1}+24 t_{1}^{2}+15}{6 t_{1}^{2}} \pm \frac{1}{6 t_{1}^{2}}\left[4\left(t_{1}^{4}-t_{1}^{3}-t_{1}+1\right) c_{1}^{2}+\right. \\
& \left.+6\left(8 t_{1}^{3}-16 t_{1}^{4}+13 t_{1}^{2}+5 t_{1}-10\right) c_{1}+9\left(64 t_{1}^{4}-40 t_{1}^{2}+25\right)\right]^{\frac{1}{2}}
\end{aligned}
$$

с соответствуюшими асимптотиками при $c_{1} \rightarrow \pm \infty$ :

$$
c_{2}^{ \pm}\left(c_{1} ; m_{1}\right) \sim \alpha^{ \pm} c_{1}, \quad \alpha^{ \pm} \equiv \frac{1}{6 t_{1}^{2}}\left(\left(t_{1}-2 t_{1}^{2}-2\right) \pm 2\left(1-t_{1}\right) \sqrt{t_{1}^{2}+t_{1}+1}\right) .
$$

Найденное семейство (59)-(62) всех решений уравнения (58) обладает особыми свойствами: $c_{2}^{+}\left(20 ; m_{1}\right)=c_{2}^{-}\left(6 ; m_{1}\right)=0$ при всех $m_{1}$, но значения $c_{2}^{ \pm}$при $c_{1}=0$ зависят от $m_{1}$ :

$$
c_{2}^{ \pm}\left(0 ; m_{1}\right)=\frac{1}{6 t_{1}^{2}}\left(24 t_{1}^{2}+15 \pm 3\left(64 t_{1}^{4}-40 t_{1}^{2}+25\right)^{\frac{1}{2}}\right) .
$$

Благодаря этим свойствам семейства и его непрерьвности по параметру $m_{1}$ при любых $c_{1}$ в координатной плоскости $\left(c_{1}, c_{2}\right)$ графики всех решений $c_{2}^{-}$пересекают ось абсцисс в точке $(6,0)$, а графикики всех решений $c_{2}^{+}-$в точке $(20,0)$, график 


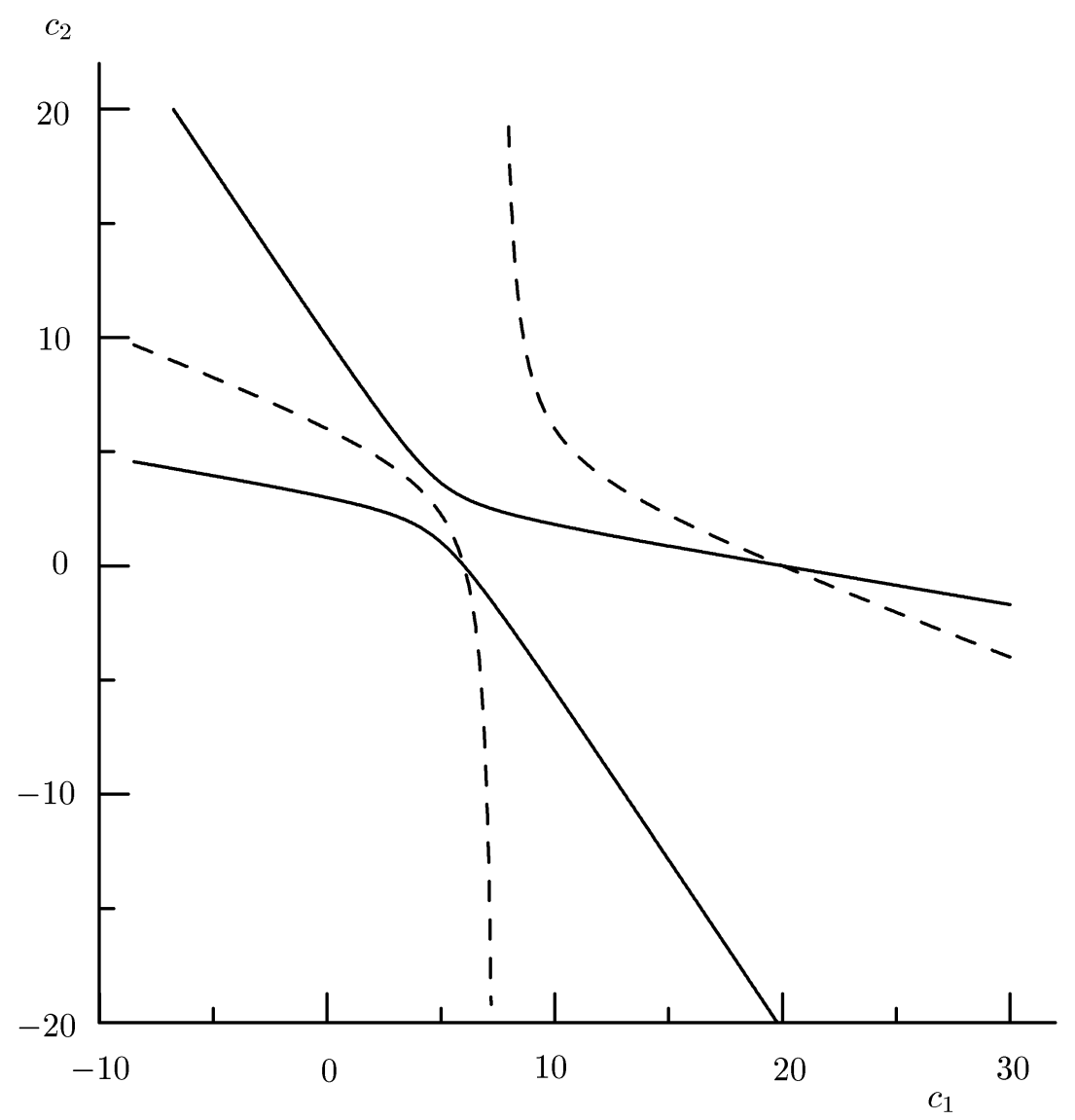

Рис. 2. Функции $c_{2}^{ \pm}\left(c_{1} ; m_{1}\right)$ при $m_{1}=0$ (сплошные линии) и при $m_{1}=\infty$ (штриховые).

решения $c_{2}^{+}\left(c_{1} ; m_{1}\right)$ или $c_{2}^{-}\left(c_{1} ; m_{1}\right)$ при любом $m_{1} \in(0, \infty)$ проходит между графиками соответствуюших предельных решений $c_{2}^{ \pm}\left(c_{1} ; 0\right)$ и $c_{2}^{ \pm}\left(c_{1}, \infty\right)$ (см. рис. 2$)$.

В рассматриваемом случае, в отличие от предыдущего случая трех разных частиц, выражения для коэффициентов $B_{n}^{i}$ рядов (45) настолько компактные, что их стоит вывести в явном виде. При $c_{2}=c_{2}^{ \pm}$оба уравнения двумерной системы $(54)$, т.е. системы

$$
\left(\mathbf{I}-\mathbf{D}^{2} \mathbf{D}^{1}\right) \mathbf{X}_{1}=0
$$

линейно зависимы. Поэтому элемент $X_{1}^{1}$ искомого столбца $\mathbf{X}_{1}=\left(X_{1}^{1}, X_{1}^{2}\right)^{T}$ можно задать любым числом, кроме нуля, затем исключить из этой системы первое уравнение, а второе разрешить относительно $X_{1}^{2}$. Потом по формулам (53) нетрудно найти столбец $\mathbf{X}_{2}=-\mathbf{D}^{1} \mathbf{X}_{1}$, затем вычислить столбец $\mathbf{X}_{3}$ как решение системы (48) при $m=3$ и, наконец, с помощью соотношений (46) определить все коэффициенты $B_{n}^{i}$. При выборе $X_{1}^{1}=-8$ получаются два набора $B_{n}^{i+}$ и $B_{n}^{i-}$ коэффишиентов $B_{n}^{i}$, отвечаюших решениям 
$c_{2}=c_{2}^{ \pm}$уравнения (58), причем $B_{1}^{1 \pm}=-1$, a

$$
\begin{aligned}
B_{1}^{2 \pm} & =\frac{c_{2}^{ \pm}}{2 f^{ \pm}}\left(c_{1}-10+2 t_{1}\left(t_{1} c_{2}^{ \pm}-2\right)\right), \\
B_{2}^{1 \pm} & =\frac{4}{5 f^{ \pm}}\left(10\left(6-c_{2}^{ \pm}\right)+t_{1}\left(c_{1}+2 t_{1}\left(c_{2}^{ \pm}-8\right)\right) c_{2}^{ \pm}\right), \\
B_{2}^{2 \pm} & =\frac{2 c_{2}^{ \pm}}{5 f^{ \pm}}\left(10-c_{1}-2 t_{1}\left(c_{2}^{ \pm}-8\right)\right), \\
B_{3}^{1 \pm} & =\frac{15}{8 g^{ \pm}}\left(\left(8 t_{1}^{2}\left(t_{1}^{2}-1\right)+3\right) c_{2}^{ \pm} B_{2}^{1 \pm}+\left(1-4 t_{1}^{2}\right) c_{1} B_{2}^{2 \pm}\right), \\
B_{3}^{2 \pm} & =\frac{15}{16 g^{ \pm}}\left(\left(1-4 t_{1}^{2}\right) c_{2}^{ \pm} B_{2}^{1 \pm}+3 c_{1} B_{2}^{2 \pm}\right) ; \\
f^{ \pm} & \equiv\left(c_{1}-10\right)\left(c_{2}^{ \pm}-6\right)+2 t_{1}^{2} c_{2}^{ \pm}\left(c_{2}^{ \pm}-8\right), \quad g^{ \pm} \equiv\left(1-t_{1}^{2}\right)^{2} c_{1} c_{2}^{ \pm} .
\end{aligned}
$$

Как было показано, при любых массах и для любого значения $c_{1}$ найдутся два значения $c_{2}=c_{3}=c_{2}^{ \pm}$такие, что соответствуюшие им две функции (44) с индексом $m=3$ и коэффициентами $B_{n}^{1 \pm}$ будут точными решениями уравнения Шредингера (21). Так как в асимптотиках (63) коэффициенты пропорциональности $\alpha^{ \pm}$отрицательные, то такие регулярные решения сушествуют даже при сколь угодно сильном притяжении $\left(c_{1} \rightarrow\right.$ $-\infty)$ в паре частиц $p_{2}, p_{3}$ и соответствуюшем ему достаточно сильном отталкивании $\left(c_{2}=c_{3}=c_{2}^{ \pm} \sim \alpha^{ \pm} c_{1}>0\right)$ в двух других парах частиц. Следовательно, даже если условие (1) при $c=c_{1}$ и $b=0$ не вьполняется $\left(c_{1}<-1 / 4\right)$ и два бозона $p_{2}$ и $p_{3}$ в отсутствие третьей частицы $p_{1}$ "падают друг на друга", то в трехчастичной системе, содержашей эти же бозоны, их "падение" в состоянии (44) предотврашается отталкивающими взаимодействиями с третьей частищей.

4.3. Три тождественных бозона. Теперь $m_{k}=1$ и $c_{k}=c_{2}$ при всех $k=1,2,3$. Поэтому характеристическое уравнение (55) имеет вид $c_{2}-4=0$. Его корню $c_{2}=4$ отвечают коэффициенты $B_{1}^{1}=1, B_{2}^{1}=-4 / 5$ и $B_{3}^{1}=-1$.

Случай трех тождественных бозонов подробно описан в работе [5]. Дополним ее важными замечаниями, справедливыми при любом $t=2(m-1)$.

Матрища $\mathbf{A}^{t}$ системы (49) является трехдиагональной и симметричной матрицей с диагональными элементами (52). Эта матрица имеет диагональное преобладание, если $c_{2} \leqslant 0$. Поэтому все корни $c_{2}^{1}, \ldots, c_{2}^{m-1}$ характеристического уравнения (55) действительные и положительные.

Если, последовательно полагая $n=1,2, \ldots, m$, вычислять детерминанты $d_{n}$ главных миноров порядка $n$ матрицы $\mathbf{A}^{t}$ разложением по элементам их последних строк, то получится рекуррентная цепочка равенств

$d_{1}=\mathbf{D}^{1}, \quad d_{2}=\mathbf{D}^{2} d_{1}-1, \quad d_{n}=\mathbf{D}^{n} d_{n-1}-d_{n-2}, \quad n=3, \ldots, m-1 ; \quad \operatorname{det} \mathbf{A}^{t}=d_{m-1}$,

согласно которой $d_{m-2} \neq 0$, если $\operatorname{det} \mathbf{A}^{t}=0$. Следовательно, только два уравнения системы (49) для искомых коэффициентов $X_{n}^{1}$ могут быть линейно зависимыми. Значит, все корни $c_{2}^{1}, \ldots, c_{2}^{m-1}$ разные. 
Итак, если в системе трех тождественных бозонов взаимодействия притягивающие $\left(c_{2}<0\right)$, то функция типа (44) не может быть точным решением уравнения Шредингера $(21)$, а в противном случае для любого $t=4,6, \ldots$ сушествуют $m-1=t / 2$ разных значений $c_{2}>0$, при которых такие функции - точные решения.

\section{5. ЗАКЛЮЧЕНИЕ}

Основной результат работы - доказательство критерия сушествования точных решений (19), (20) уравнения Шредингера (21) с нецентральными взаимодействиями (7) и полное описание метода построения этих решений и их фаддеевских компонент. Последние можно использовать как эталонные для тестов алгоритмов численного решения уравнений Фаддеева.

Основные физические выводы таковы. Все решения (2) двухчастичного уравнения Шредингера с потенциалом центробежного типа сингулярны в точке парного столкновения. Присутствие третьей частищы радикально изменяет ситуацию: при условиях (37) сушествуют всюду регулярные решения трехчастичного уравнения Шредингера с парными взаимодействиями того же типа. Из примеров следует, что даже если условие "падения" выполняется в выбранной паре частиц, то для системы трех частиц при вполне определенных параметрах взаимодействий в других парах частиц такого "падения" не происходит.

\section{Список литературы}

[1] Л. Д. Ландау, Е. М. Лифииц. Квантовая механика. М.: Наука, 1974.

[2] С. П. Меркурьев, Л. Д. Фаддеев. Квантовая теория рассеяния для систем нескольких частиц. М.: Наука, 1985.

[3] В. В. Пупьиев. ЭЧАЯ. 1999. Т. 30. С. 1562.

[4] Y. Avishai. J. Math. Phys. 1975. V. 16. P. 1491.

[5] V. V. Pupyshev. Phys. Lett. A. 1989. V. 140. P. 151.

[6] Р. И. Джсибути, Н. Б. Крупенникова. Метод гиперсферических функций в квантовой механике нескольких тел. Тбилиси: Мецниереба, 1984.

[7] Н. Я. Виленкин. Специальные функции и теория представления групп. М.: Наука, 1965.

[8] B. В. Пупьишев. ЯФ. 1999. Т. 62. С. 1955.

[9] J. Raynal, J. Revai. Nuovo Cimento. A. 1970. V. 68. P. 612.

[10] Э. Камке. Справочник по обыкновенным дифференциальным уравнениям. М.: Наука, 1976.

[11] М. Абрамовиц, И. Стиган. Справочник по специальным функциям. М.: Наука, 1979.

[12] А. П. Прудников, Ю.А. Брычков, О. И. Маричев. Интегралы и ряды. Специальные функции. М.: Наука, 1983. С. 581.

[13] П. Ланкастер. Теория матриц. М.: Наука, 1978.

[14] В. В. Воеводин. Вычислительные основы линейной алгебры. М.: Наука, 1977.

[15] В. В. Пупыщев. Физические и ложные слагаемые центральных парных взаимодействий. Препринт Р4-2000-136. Дубна: ОИЯИ, 2000. 